Espacio, Tiempo y Forma, Serie II, Historia Antigua, t. 23, 2010, págs. 519-540

\title{
La Romanización de Mauretania Tingitana (Marruecos)
}

\author{
The Romanization of Mauretania Tingitana (Morocco)
}

\author{
EnRIQUe Gozalbes Cravioto*
}

\begin{abstract}
RESUMEN ABSTRACT
Se estudian tres aspectos principales: la evolución de los estudios y conclusiones en relación con la romanización de Marruecos, la existencia de la relación entre la población urbana y la población con organización tribal, y finalmente, se realiza un balance acerca del fenómeno de la romanización en esta provincia del Imperio.

In these work three principal aspects are studied: the evolution of the studies and conclusions in relation with the romanization of Morocco, the existence of the relation between the urban population and the population with tribal organization, and finally, realizes a balance sheet brings over of the phenomenon of the romanización in this province of the Empire.
\end{abstract}

PALABRAS CLAVE: Indigenismo, transformación, África romana, economía, arqueología.

KEYWORDS:

Indigenous, transformation, Roman Africa, economy, archaeology

El estudio histórico de un proceso de la importancia de la romanización es inseparable del contexto en el que se realiza dicha investigación. La romanización tiene ya una particularmente extensa tradición investigadora que refleja los avatares del conocimiento sobre la antigüedad desde hace más de un siglo, con los correspondientes posicionamientos ideológicos adoptados por los historiadores en

* Universidad de Castilla-La Mancha, Avda de los Alfares 44, 16002 Cuenca. E-Mail: Enrique.Gozalbes@uclm.es 
cada momento ${ }^{1}$. La importancia de la ocupación romana en el Norte de África, por las evidencias de sus construcciones más monumentales, de las obras de arte, por los testimonios de la literatura latina del África, por la propagación del cristianismo y la importancia de la iglesia primitiva africana, con sus numerosos obispados, también con su producción literaria, justifica el que la romanización tenga en el Magreb una larga tradición de estudios y, que de hecho, del mismo surgiera incluso el cuestionamiento del concepto de romanización, en la medida en la que se pretendía un análisis más crítico y «descolonizador»².

Por otra parte, la propia romanización encierra unos contenidos muy determinados en relación con el Magreb, un conjunto de países en los que históricamente, desde hace muchos siglos, está impuesta otra tradición cultural, la arabo-islámica, que la sustituyó de forma bastante contundente a partir de los siglos VII-VIII. Por esta razón no puede sorprender que el África romana continúe atrayendo la atención de los investigadores, como puede observarse en la bibliografía más reciente $^{3}$, y en muchas ocasiones incluso la fascinación por sus brillantes vestigios materiales y culturales. En esta línea, el concepto de romanización como proceso histórico de larga duración aporta, sin duda, un termómetro de la evolución de la civilización clásica, en un territorio como el de la Mauretania Tingitana, incorporado al Imperio en fechas tardías (época de Claudio), si bien es cierto que la romanización se había iniciado en el mismo con anterioridad, en época de la monarquía de luba II y Ptolomeo.

Quizás el principal debate sobre la Historia del Magreb se produjo precisamente en su día, en la época colonial, en torno a si existió realmente o no un fracaso de la romanización en el Norte de África, o bien que lo que aconteció fue un simple triunfo de la islamización algunos siglos más adelante ${ }^{4}$. La cuestión que

1 El término mismo de «romanización» ha sido puesto en cuestión por los contenidos apologéticos de los que se dotó al mismo. En los últimos años los planteamientos de la historiografía plantean la relación de la romanización con el imperialismo romano; MATTINGLY, D. J. (Ed.), Dialogues in Roman Imperialism: Power, discourse and discrepant experience in the Roman Empire, Porsmouth, 1997; HINGLEY, R., «The legacy of Rome: the rise, decline and fall of the theory of Romanization», en WEBSTER, J. y COOPER, N. (Eds.), Roman Imperialism: post-colonial perspectives, Leicester, 1996, pp. 35-48. Vid. también BLÁZQUEZ, J. M. y ARCE, J. (Eds.), La Romanización en Occidente, Madrid, 1996. Vid. LE ROUX, P., «La Romanisation en question», Annales Histoire, Sciences Sociales, 59 (2), 2004, pp. 287311.

2 PFLAUM, H. G., «La romanisation de l' Afrique», Vestigia Antiquitatis, 17, 1972, pp. 53-72, reproducido en su obra Afrique Romain. Scripta Varia, I, Paris, 1978, pp. 375-392.

3 CHERRY, D., Frontier and Society in Roman North Africa, Oxford, 1998; SEBAï, M., «La romanisation en Afrique, retour sur un débat», Afrique \& Histoire, 3, 2005, pp. 39-56 ; CHRISTOL, M., Regards sur l' Afrique romaine, Paris, 2005 ; GUIRAUD, H. (Ed.), L'Afrique romaine : 1 siècle avant J. C.- début Ve siècle. Actes du Colloque, Toulouse, 2005 ; CABOURET, B. (Coord.), L' Afrique romaine : de 69 à 439, romanisation et christianisation, Paris, 2005 ; LE BOHEC, Y., Histoire de l' Afrique romaine (146 avant J. C.-439 après J. C.), Paris, 2005 ; BRIAND-PONSART, C., L' Afrique romaine, de l' Atlantique a la Tripolitaine, 146 av. J. C.- 533 ap. J. C., Paris, 2005 ; IBBA, A. y TRAINA, G., L'Afrique romaine de l' Atlantique à la Tripolitaine (69-439 ap. J. C.), Paris, 2006.

4 COURTOIS, C., Les Vandales et l' Afrique, Paris, 1955 planteó la tesis del fracaso de la romanización, por el contrario PICARD, G. C., La civilisation de l' Afrique romaine, Paris, 1959, defendió que no puede hablarse de fracaso de la pujante romanización, por el contrario, la desaparición de la tradición latina se produciría tan sólo debido al triunfo medieval de la arabización e islamización. Por su parte 
apuntamos no puede alejarse del propio hecho de que las antigüedades constituyeron la ciencia mimada de la administración colonial, en la medida en la que la ligazón psicológica de los colonizadores se planteaba con la acción de Roma, y el hecho de reflexionar acerca de la misma era una forma de hacerlo también acerca del presente.

Por otra parte, debido a la lejanía de los magrebíes respecto a la Historia Antigua esta discusión quedó olvidada después de la independencia de los países del Magreb, cuando la antigüedad quedó reducida a una limitada actividad de arqueología, de recuperación de materiales, con vistas a completar las exposiciones de los Museos, en especial cara a los turistas europeos. Por esta razón no puede extrañarnos que el revulsivo principal en los estudios sobre la romanización del Magreb, dos décadas después de su abandono, se produjera con la introducción del concepto de resistencia a la misma, que en esa estela de discusión se extendió a otras áreas regionales ${ }^{5}$.

\section{LA TINGITANA, ENTRE LA OCUPACIÓN MILITAR Y LAS BELLAS ARTES}

Cuando los colonos franceses en el Norte de África, primero sobre todo en Argelia desde el siglo XIX, pero más tarde también en Marruecos y en Túnez en el siglo XX, comenzaron a encontrar vestigios romanos en los territorios poco cultivados, no pudieron menos que mostrar una cierta emoción y continuidad psicológica con la antigua Roma. La simpatía de los investigadores europeos hacia el mundo romano era perfectamente comprensible, y también la visión de la colonización francesa (subsidiariamente española en el Norte de Marruecos) como la que venía a traer civilización y progreso donde se consideraba que se había perdido con la caída romana.

Los modelos teóricos que podían aportarse para el análisis se encontraban insertos en la visión de la superioridad europea, así como en la creencia de una tradicional resistencia de los habitantes del Norte de África a la civilización mediterránea y urbana. La historiografía sobre el Norte de África no ha podido menos que mostrar el sentido de la contraposición sedentarismo/nomadismo, y ruralidad/civilidad, que de hecho ya había sido planteada en el siglo XIV para esta

CAMPS, G., «Comment la Berbérie est devenue le Maghreb arabe», Revue de l' Occident Musulman et de la Méditerranée, 35, 1973, pp. 7-24, no aceptaba a nivel global que se produjera un fracaso de la romanización, aunque exponía que la intensidad de la misma era muy diferente, más potente en la zona oriental (Tunez) y mucho más débil en la occidental (Marruecos).

5 BÉNABOU, M., La resistance africaine a la romanisation, Paris, 1976. Y en esa misma época justamente constituyó el tema central al que se dedicó el Congreso Internacional de Estudios Clásicos; Assimilation et résistance á la culture gréco-romaine dans le monde ancien. VI Congrès International d' Etudes Classiques, 2 vols., Paris, 1976. Vid. sobre todo THÉBERT, Y., «Romanisation et déromanisation en Afrique : histoire décolonisée ou histoire inversée ?», Annales E.S.C., 33 (1), 1978, pp. 64-82, la contestación del propio BENABOU, M., «Les Romains ont-ils conquis I’Afrique», pp. 83-88, y el planteamiento de LEVAU, P., «La situation coloniale de l' Afrique romaine», pp. 89-92. Vid. GOZALBES, E., «Algunas notas acerca de la bibliografía sobre la resistencia a la romanización en el Norte de África», Tempvs, 7, 1994, pp. 33-43. 
misma zona por parte del escritor tunecino Ibn Jaldun ${ }^{6}$, en unos momentos en los que la irrupción de poblaciones nómadas había puesto en jaque las características urbanas del Magreb. Y ello es así porque el sabio tunecino supo realizar una lectura acerca de constantes profundas que afectaban al devenir de la civilización en el Magreb, y que él mismo extendió hasta los tiempos ante-islámicos.

Los vestigios arqueológicos que aparecían eran sobre todo los de ciudades, pues en muchos casos los árabes habían trasladado sus asentamientos a otros lugares, así como de unas estructuras que, en gran medida, eran interpretadas como establecimientos militares. En el caso de Marruecos, los primeros estudios del diplomático francés Charles Tissot, entre 1872 y 1875, estuvieron muy limitados por la inseguridad, que le impidieron alejarse mucho de los principales caminos, pero permitieron documentar por vez primera una propuesta de identificación de los centros romanos en el territorio ${ }^{7}$, a partir de los datos básicos del Itinerario de Antonino. Pocos años más tarde, pero aún antes de la ocupación franco-hispana de Marruecos en el Protectorado establecido en el año 1912, otro diplomático francés, Henry de la Martinière, realizó prospecciones más sistemáticas en zonas algo más amplias, con sus trabajos que presentó a la Académie des Inscriptions y también al Comité des Travaux Historiques, reflejo de un trabajo que ya era menos mera iniciativa personal ${ }^{8}$. Y además, el diplomático realizó excavaciones en las ciudades antiguas de Volubilis y Lixus.

La información de Martinière, como única disponible en ese momento, fue la que pasó al estudioso René Cagnat, en su trabajo de conjunto acerca del ejército romano en el Norte de África. Éste realizó una curiosa reinterpretación de los datos, de forma que desplazó muy hacia el Este buena parte de los asentamientos romanos no bien conocidos, en concreto ubicó en esa zona ciudades como Babba, Vopisciana, Gilda o Prisciana, y también planteaba la existencia de una Fez romana. Estos indicios arqueológicos se llevaron al orden de la ocupación militar, en los momentos de establecimiento del Protectorado franco-hispano en Marruecos, y este fue el uso que hizo de ellos en su interpretación René Cagnat. Su visión del conjunto del Magreb, y muy en especial de Marruecos, era de una zona en la que los problemas de orden y levantamientos eran continuos, y exigían la actuación permanente del ejército ${ }^{9}$. $Y$ en el caso de Marruecos esos problemas serían especialmente importantes, pues en la re-

6 IBN JALDUN, Introducción a la Historia Universal (Al-Muqaddimah), México, 1997. Vid. los estudios de LACOSTE, Y, ., Ibn Khaldoun. Naissance de l' histoire passée du Tiers Monde, Paris, 1966 ; BAALI, F., Society, State, and Urbanism. Ibn Khaldun's Sociological Thought, New York, 1988 ; CHABANE, D., La pensée de l'urbanisation chez Ibn Khaldûn (1332-1406), Paris, 2000.

7 Realizada en la extensa Memoria de TISSOT, C., Recherches sur la géographie comparée de la Maurétanie Tingitane, Paris, 1878. Vid. el análisis de REBUFFAT, R., «Histoire de I' identification des sites urbains antiques du Maroc», L'Africa Romana, XIII, Roma, 2000, pp. 865-914.

8 MARTINIĖRE, H. de la, Souvenirs du Maroc. Voyages et Missions, Paris, 1920, donde destaca la descripción de sus excavaciones en Volubilis y en Lixus. Por otra parte fue el autor del primer estudio de Historia Antigua de Marruecos; «Esquisse de l' histoire du Maroc avant l' arrivée des Arabes», Bulletin du Comité des Travaux Historiques, 1912, pp. 142-184.

9 CAGNAT, R., L'Armée romaine d'Afrique, 2 vols., Paris, 1912. 
construcción que se realizaba, y que ha tenido trascendencia hasta el momento actual, las transformaciones de la romanización disminuían en sentido EsteOeste, eran potentes en Tunez, desiguales en Argelia, y relativamente débiles en Marruecos ${ }^{10}$.

El inicio del Protectorado francés en Marruecos coincidió, a partir del año 1916 en concreto, también con el comienzo de las excavaciones arqueológicas en Volubilis que serían desarrolladas por Louis Chatelain. El investigador francés utilizó para la limpieza y comienzo de la restauración de la ciudad romana a los prisioneros alemanes de la Primera Guerra Mundial, pero en cualquier caso, durante poco más de dos décadas desarrolló trabajos importantes en la ciudad y en otros lugares ${ }^{11}$. Producto de ello fue la realización de su Tesis en el que, a partir de los vestigios, matizaba la importancia del componente militar, señalando también los avances del urbanismo y de la vida romana en Marruecos, con magníficos ejemplos de mosaicos y bellas obras de $A_{r t e}{ }^{12}$. Debe indicarse que la arqueología ha mostrado este mismo tipo de vida urbana, con casas lujosas, en otras ciudades del Marruecos antiguo, en concreto Lixus, Banasa o Tingi.

En los años treinta del siglo XX Jerôme Carcopino, quien se declaraba alumno y seguidor de Stéphan Gsell autor de la monumental Histoire Ancienne de l' Afrique du Nord (8 tomos, Paris, 1914-1928), intentó completar los datos del análisis respecto a Marruecos. A su juicio las obras de arte de Volubilis habían sido recibidas con un entusiasmo a todas luces excesivo. Juntando una buena parte de sus trabajos, y escribiendo algunos para la ocasión, redactó lo que en buena parte pretendía ser la primera Historia de Marruecos en los tiempos antiguos ${ }^{13}$. Basta con revisar la obra de Carcopino para descubrir en la misma no ya a un estudioso de las Bellas Artes, especialista en epigrafía como era el caso de Chatelain, sino a un verdadero historiador de la antigüedad, más allá de los análisis de carácter ideológico sobre su aportación.

Su análisis sobre el Marruecos romano fue menos optimista que el de Chatelain, en la medida en la que consideró que Roma no fue, naturalmente, nada más que a lo suyo, en busca de su propio interés, en los gustos, en la explotación de los recursos, extinguiendo los elefantes, o desforestando amplias superficies de los montes. No obstante, la provincia romana de la Tingitana significó avances en la

10 MESNAGE, J., La Romanisation de l' Afrique: Tunisie, Algérie, Maroc, Paris, 1913.

11 EN NACHIOUI, E. A., «Las primeras excavaciones en Volubilis (Marruecos) ¿Arqueología, historia o simple colonización?», Pyrenae, 26, 1995, pp. 161-170. Sobre los estudios arqueológicos en la zona española de Marruecos, GOZALBES, E., «Los primeros pasos de la arqueología en el Norte de Marruecos", en BERNAL, D. y otros (Coords.), En la orilla africana del Círculo del Estrecho: historiografía y proyectos actuales, Cádiz-Tetuán, 2008, pp. 33-62. Destacará en 1921-1922 la localización y estudio, el primero realizado, de un castellum militar romano en Tamuda (Tetuán).

12 CHATELAIN, Le Maroc des Romains: etude sur les centres antiques de la Mauritanie occidentale, Paris, 1944; reed., 1968. En el terreno de la Historia Antigua puede verse su puesta a punto «Le Maroc avant l' islam", Encyclopédie coloniale et maritime. Le Maroc, Paris, 1940, pp. 76-88. Un listado de la bibliografía de Chatelain sobre el Marruecos romano puede verse en BROUQUIER-REDÉ, V. y LENOIR, E., «Bibliographie du Maroc Antique», L’ Africa Romana, XIII, Roma, 2000, pp. 1005-1010.

13 CARCOPINO, J., Le Maroc Antique, Paris, 1943. 
urbanización del territorio, en concreto en lo que consideraba el Marruecos «útil» (siguiendo la terminología colonialista de Lyautey): les activités de la Maurétanie Tingitane reçurent alors une impulsión nouvelle. Toutes les cultures que les puniques y avaient introduites ou encouragées et qui avaient continué de prospérer sous les rois maures prirent un essor acru ${ }^{14}$.

No obstante, las limitaciones de la política romana se dejaron sentir en la medida en la que Carcopino valoraba: ils ont enfermé leur exploitation dans les limites étroites que par souci de sécurité ils avaient tracées à leur colonie de peuplement ${ }^{15}$. De hecho, sous le Haut-Empire, cette politique, en excluant toute pénétration véritable, devait, à l' expérience, se réveler inefficace.... En fait, la Romanisation de Volubilis ou de Banasa, deux centres du Maroc Antique, avait précedé la conquête romaine au lieu d" en découler, et ailleurs elle semble bien ne s" etre accomplie qu' avec une troublante lenteur ${ }^{16}$.

Así pues, Roma mantuvo de forma menos sólida el control en su provincia de lo que lo habían hecho los reyes (luba II y Ptolomeo) puestos por ellos previamente, de forma que los levantamientos indígenas fueron frecuentes: dans ces conditions, avec perpétuelles agitations et cette insécurité généralissée, il est natural que Dioclétien, lorsqu' il s' est rendu compte qu' il ne pouvait défendre le front de l' empire qu' en le raccourcissant, $n$ ' ait point hésité à abandonner à son sort, en 285 , une bonne moitié des Maurétanies ${ }^{17}$. En todo caso, cabe indicar que la visión de «inseguridad generalizada», derivada de hechos de su época (como la lucha de Abdelkrim) en su mayor parte no es aceptada por la mayor parte de los investigadores; por el contrario, su observación acerca de la limitación de la colonización romana plantea hoy día una sugestiva reflexión.

El sucesor de Chatelain en la Dirección de Antigüedades del Protectorado francés, Raymond Thouvenot, en gran parte continuó las actividades y planteamientos de su predecesor. En principio se centró sobre todo en las excavaciones de Volubilis, pero también sobre todo en las de la colonia romana de lulia Valentia Banasa. En cualquier caso, junto con las obras de Arte, y las inscripciones, introdujo otros elementos que son más puramente arqueológicos modernos, tales como el estudio de las cerámicas, de las ánforas y de las monedas. En distinto orden, en Banasa en sus excavaciones aparecieron una gran cantidad de planchas con diplomas militares, de tal forma que el autor pudo pormenorizar la importante ocupación militar de la provincia ${ }^{18}$.

14 CARCOPINO, J., p. 40.

15 CARCOPINO, J., p. 41.

16 CARCOPINO, J., pp. 43-44.

17 CARCOPINO, J., p. 45.

18 Una relación completa de las publicaciones de Raymond Thouvenot sobre Marruecos puede verse en BROUQUIER-REDDÉ, V. y LENOIR, E., «Bibliographie», pp. 1061-1069. Al igual que Carcopino, Raymond Thouvenot se caracteriza por partir de la arqueología para hacer reconstrucción histórica, más allá de la mayor o menor validez de estas conclusiones históricas en relación con el avance posterior de las investigaciones. 
Muchísimos años después de su abandono de Marruecos y jubilación, Thouvenot publicaba su último trabajo sobre Marruecos, dedicado al urbanismo romano ${ }^{19}$. En el mismo destacaba que los romanos se establecieron en un país que previamente ya disponía de vida urbano, pero sólo ocuparon una zona muy limitada, ce que le Maréchal Lyautey appelait le Maroc utile. Le climat y est moins rude, tempéré par la brise de mer qui fait sentir son effet jusqu' au delà de Fès. Les pluies tombent en suffisance en noviembre pour les semailles, en février pour la croissance des céréales. Les vallées et le plateau du Rharb sont couvertes d' une épaisse couche d' alluvions fértiles. Sur les pentes on peut cultiver la vigne, l' olivier, le figuier ${ }^{20}$. En cualquier caso, Thouvenot introducía ya aquí una visión que rebasaba el ámbito de lo militar y defensivo, si bien en momentos en los que había cambiado la lectura historiográfica al respecto, con el desarrollo de lo que se ha llamado «descolonización de la Historia Antigua norteafricana».

\section{ENTRE LA ARQUEOLOGÍA Y LA ECONOMÍA ANTIGUA}

En este sentido, debemos destacar la importancia que en relación con el Marruecos romano tuvieron los estudios de Miguel Tarradell Mateu. Inspector de Arqueología del Protectorado Español en el Norte de Marruecos, y Director del Museo Arqueológico de Tetuán, desde 1948 a 1956, su aportación se dejó sentir en múltiples aspectos. No obstante, uno de ellos fue, sin duda, su capacidad para trascender de la pura arqueología para construir Historia Antigua ${ }^{21}$. Tarradell fue autor de una extensísima producción sobre el Marruecos Antiguo, con la que abrió muchas rutas al conocimiento, a partir de la lectura de los procesos históricos representados por los objetos materiales ${ }^{22}$. En este sentido, más allá de las obras de arte romanas halladas sobre todo en las excavaciones de Lixus, como los grupos de Hércules y Anteo y Teseo y el Minotauro, o los magníficos ejemplos de mosaicos de las viviendas, algunos de ellos instalados en el Museo de Tetuán, los hallazgos de centros más apartados y modestos respecto a las ciudades, a juicio de Tarradell eran bastante significativos.

En lo que respecta a las cuestiones que ahora nos ocupan, destaca su trabajo acerca de las etapas de la romanización de Marruecos, en el que planteaba la existencia de un inicio con anterioridad a la propia conquista, en especial con la política puesta en práctica por Augusto, y un desarrollo mayor iniciado desde comienzos del

19 THOUVENOT, R., «L' urbanisme romain dans le Maroc Antique», Revista de la Universidad Complutense, 112 (=Homenaje a García Bellido IV), 1979, pp. 325-349.

20 THOUVENOT, R., p. 327.

21 Tal y como hemos reseñado y destacado en nuestro análisis; GOZALBES, E., «África antigua en la historiografía y arqueología de época franquista», en ÁLVAREZ, M. y WULFF, F. (Coords.), Antigüedad y franquismo (1936-1975), Málaga, 2003, pp. 135-160. Vid.también GOZALBES, E y PARODI, M., «Miguel Tarradell y la arqueología del Norte de Marruecos», en BERNAL, D. y otros, Arqueología y Turismo en el Círculo del Estrecho. III Seminario Hispano-Marroquí de especialización en Arqueología, Cádiz, 2011, pp. 104-127.

22 La extensa producción de Tarradell aparece listada en BROUQUIER-REDDË, V. y LENOIR, V., pp. 1058-1061. 
siglo II; Tarradell detectaba la existencia de la fuerte crisis del siglo III, que él creía reconocer en posibles niveles de destrucción en diversos centros del Marruecos romano septentrional, así como en la inestabilidad representada por los ocultamientos de tesorillos; finalmente, Tarradell señalaba que el repliegue romano a partir de Diocleciano convirtió la Tingitana en una especie de defensa avanzada de Hispania, pero que resultaba indudable el desarrollo de la península Noroeste en esa época ${ }^{23}$.

Pero la gran aportación de Tarradell en relación con el Marruecos romano fue la «pacificación» de los restos ${ }^{24}$. El mismo autor indicaba como hasta ese momento los estudiosos de la arqueología, en cuanto salían de las ciudades romanas, tenían tendencia a encontrar en todos los centros fortines y lugares estratégicos. Sin embargo, el análisis de una buena parte de estas instalaciones apuntaban en un sentido distinto, así muchas de ellas se interpretaban mejor como granjas o villas rústicas, y en el caso de las costas como instalaciones de fabricación del salazón de pescado: más que lugares fortificados y emplazamientos de guarniciones, nos encontramos con ciudades y con explotaciones agrícolas e industriales ${ }^{25}$.

Después de 1956, con la recuperación de la independencia de Marruecos, los estudios arqueológicos continuarán con el Service d" Archéologie, al frente del cual estará durante varios años Maurice Euzennat. Sus estudios tratarán de rebasar el ámbito de las ciudades romanas principales, con un análisis de asentamientos rurales, pero sobre todo de los centros militares. Los trabajos de Euzennat mostrarán que, pese a las dudas, y que no constituyera la totalidad de la verdad, lo cierto es que la ocupación militar de la Mauretania Tingitana tuvo gran importancia, en la medida sobre todo en la que se precisaba de frenar la irrupción de poblaciones nómadas procedentes del exterior ${ }^{26}$. En parte, estos trabajos sobre los campamentos militares romanos de la Tingitana, de forma separada, serían continuados años más tarde por parte de René Rebuffat, quien entrecruzaría la información ofrecida por otras fuentes diferentes, en especial con el uso intensivo de la epigrafía latina que ha documentado los nombres de las distintas unidades del ejército establecidas en lugares diferentes de la Tingitana.

Estudios muy diferentes, desde 1958 a 1967, serán los desarrollados en la zona de Tánger, Arcila y Larache, por parte de Michel Ponsich; sus prospecciones más sistemáticas le van a permitir detectar la fuerte densidad de la ocupación romana en algunas de las zonas estudiadas, muy en especial en todo el territorio de

23 TARRADELL, M., «Acerca de las etapas de la romanización en Marruecos», III Congreso Arqueológico Nacional, Zaragoza, 1955, pp. 213-220. Una revisión de algunos aspectos de la crisis del siglo III en relación con los tesorillos, en GOZALBES, E., «La circulación monetaria alto-imperial en el norte de la Mauretania Tingitana», ETF, Historia Antigua, 19-20, 2006-2007, pp. 211-.227.

24 TARRADELL, M., «Marruecos antiguo: nuevas perspectivas», Zephyrus, 5, 1954, pp. 105-139.

25 TARRADELL, N., «Marruecos antiguo», p. 128.

26 EUZENNAT, M., Le limes de Tingitane. La frontière meridionale, Paris, 1989. Una relación completa de publicaciones del autor sobre Marruecos en BROUQUIER-REDDÉ, V. y LENOIR, E.., pp. 1016-1019. Vid. SOUVILLE, G., «Journé d" études à la mémoire de Maurice Euzennat», Antiquités Africaines, 42, 2006, pp. 15-24 ; HALLIER, G., «Maurice Euzennat et le Maroc Antique», Antiquités Africaines, 42, 2006, pp. 45-47. 
Tánger y en las proximidades de Larache, y junto a esta fuerte ocupación territorial señalará el más que evidente predominio de los elementos productivos ${ }^{27}$. Quizás las novedades principales respecto a Tarradell vinieron representadas por la metodología de la investigación, que después aplicaría en España a la cuenca del Guadalquivir, y en la introducción de un mayor protagonismo del mundo indígena en los análisis.

En buena parte, los estudios de Tarradell y de Ponsich eran complementarios, los del primero en los años cincuenta y los del segundo en los sesenta. No obstante, a partir de la salida de Ponsich de Tánger, en la segunda mitad de los años sesenta, desapareció la investigación arqueológica sobre el mundo romano en el Norte de Marruecos, quedando con importancia aminorada en la propia Volubilis. En todo caso, cabe indicar que en estas épocas se desarrolló una arqueología muy limitada, pero con su importancia, en los estudios de Carlos Posac Mon en Ceuta; con sus estudios, la Ceuta romana iba reapareciendo con sus cerámicas, monedas, lucernas, tumbas y sarcófagos. Este nuevo punto de la antigua Tingitana estaba destinado a ofrecer novedades en el futuro ${ }^{28}$.

Curiosamente, si los años setenta, y en buena parte ochenta, son de crisis de la arqueología romana en Marruecos, sin embargo en estos momentos se produce una aportación fundamental en el desarrollo de los estudios. Nos referimos a la Tesis de Bénabou, en la que focalizaba la mirada en lo que llamaba «resistencia africana a la romanización». Bénabou trataba de superar la visión achacada a la época colonial acerca de la existencia de un dualismo de población, romanizado o no romanizado. A su juicio, había un componente muy importante que no había sido destacado, el de los romanizados de una forma parcial, y que en su actitud alternativa, a favor de la transformación, o resistiendo a la misma, estuvo la clave del llamado «fracaso de la romanización» en Marruecos y en otros países del Magreb. El estudio de Bénabou creó todo una estela historiográfica, en el debate sobre la resistencia y su interpretación, incluido el caso de la Tingitana interpretado desde la historiografía anglosajona como una «sociedad de frontera» ${ }^{29}$.

27 La obra fundamental de Ponsich sobre Marruecos es su Tesis Doctoral; PONSICH, M., Recherches archéologiques a Tanger et dans sa région, Paris, 1970. El autor publicó en los años sesenta sendos trabajos muy completos referidos al Atlas archéologie du Maroc, todo el territorio entre Tánger y Larache, en dos volúmenes del Bulletin d' Archéologie Marocaine. Sobre la bibliografía de Ponsich, vid. BROUQUIER-REDDÉ,, V. y LENOIR, E., pp. 1045-1048. Sobre las aportaciones de Ponsich, vid. BLÁZQUEZ, J. M., «Tres grandes arqueólogos de Mauretania Tingitana: M. Ponsich, R. Thouvenot y M. Tarradell», L' Africa Romana, XIII, Roma, 2000, pp. 1089-1105; IDEM, «La obra de Ponsich y de Tarradell sobre Marruecos», Actas del I Seminario Hispano-Marroquí de especialización en arqueología, Cádiz, 2006, pp. 47-53.

28 POSAC MON, C., «La arqueología en Ceuta entre 1960-1970», Noticiario Arqueológico Hispánico, 15, 1971, pp. 227-235. Vid. los materiales de estas exploraciones publicados en FERNÁNDEZ SOTELO, E. A., Sala Municipal de Arqueología de Ceuta. Guía-catálogo, Ceuta, 1980.

29 GOZALBES, E., «Roma y las tribus indígenas de la Mauretania Tingitana: un análisis historiográfico", Florentia lliberritana, 3, 1992, pp. 271-302. No está de más indicar que la sugerencia acerca del papel decisivo que en el proceso tuvieron los semi-romanizados, así como la propia actitud romana al respecto, estaba ya presente en ROSTOVTZEFF, M., Historia Económica y Social del Imperio Romano, Madrid, 1973. 
El abandono de la arqueología romana tan sólo tendría la excepción de la apertura del campo de excavaciones en Dchar Jdid, donde hasta ese momento se atribuía la localización de la antigua Ad Mercuri. Los inicios del trabajo se efectuaron en 1977, pero tan sólo será a partir de 1982, y en la segunda mitad de los años ochenta, cuando los trabajos alcanzaron dimensiones importantes. Como resultado de los mismos se hallarán diversas inscripciones, reutilizadas en un recinto más tardío, que mostraban que el lugar correspondía en realidad con la colonia romana Iulia Constantia Ziliß ${ }^{\circ}$. Junto con esta importante identificación, en las excavaciones aparecieron una gran cantidad de monedas antiguas, que permiten trazar un esquema de la circulación monetaria ${ }^{31}$. La atonía en la que había caído la arqueología marroquí se remedió en esta época con la creación de un nuevo centro de formación de investigadores y de investigación, que tomaba el relevo del antiguo Service d" Archéologie, en concreto el Institut National des Sciences de l'Antiquité et du Patrimoine (INSAP).

En cualquier caso, esta etapa que se inicia a finales de los años ochenta se va a caracterizar por la entrada en liza de nuevos investigadores españoles. En 1987 se presentan en las Universidades españolas las primeras Tesis Doctorales realizadas por españoles sobre la antigua Mauretania Tingitana, en concreto la de Fernando López Pardo dedicado a un análisis general de la Historia del territorio, y la nuestra, Enrique Gozalbes, sobre la economía del reino mauritano y de la provincia romana alto-imperial ${ }^{32}$. En ambos casos, retomamos los trabajos de Tarradell y de Ponsich para tratar de profundizar en aspectos diversos, de la ocupación del territorio y de su explotación. También bastantes años más tarde la Tesis Doctoral de Lluis Pons Pujol ha venido a aportar un análisis complementario en el estudio pormenorizado de los envases anforarios ${ }^{33}$. Los mismos muestran la unidad de comercialización de muchas producciones hispanas y tiongitanas, como aceite de oliva, salazones de pescado o vino, que podían salir de forma conjunta al consumo en diversos países mediterráneos ${ }^{34}$.

\section{LAS CIUDADES Y LA SEDENTARIZACIÓN}

En casi todo lo escrito hasta el momento, y que hemos resumido en las aportaciones más emblemáticas, hay argumentaciones con datos y reflexiones que son válidos para el dossier de la romanización, más allá de que muchas contribuciones se hayan centrado en el aspecto concreto de la arqueología. El rechazo de las óp-

30 LENOIR, M., «Ab eo XXV in ora Oceani colonia Augusti lulia Constantia Zilil», L' Africa Romana. IV, Sassari, 1987, pp. 435-444.

31 DEPEYROT, G., Zilil I. Le numéraire, Paris, 1999.

32 En este segundo caso la Tesis publicó un amplio extracto; GOZALBES, E., Economía de la Mauritania Tingitana, Ceuta, 1987.

33 PONS PUJOL, L., La economía de la Mauritania Tingitana, s. I-III d C.., Barcelona, 2010.

34 Vid. también PONSICH, M., Aceite de oliva y salazones de pescadp, factores geo-económicos de Bética y Tingitana, Madrid, 1988, y nuestro análisis en GOZALBES, E., «La provincia romana de la Mauretania Tingitana, Algunas visiones actualizadas», Gerión, 28, 2010, pp. 31-51. 
ticas colonialistas ha llevado, en muchas ocasiones, a tratar con displicencia las tesis mantenidas por Jerôme Carcopino, uno de los estudiosos con perspectiva histórica, pero al menos hay una sobre la que debemos volver: las fuertes limitaciones de la actuación romana en Marruecos. Eran limitaciones de su propia política, que no pasó del interés por mantener una ocupación y una explotación puramente coloniales. La única matización posible a esta observación se encuentra posiblemente en el siglo III, momento de auge máximo primero, pero también de crisis más adelante en el caso del África romana.

La primera característica que debemos reflejar en el desarrollo de la romanización se centra en la fuerte limitación que en todo momento tuvo el desarrollo urbano. En la Tingitana existió una fuerte tradición urbana en muchos lugares, pues es cierto que la ciudad precedió en muchos siglos a la conquista romana ${ }^{35}$. Bénabou estableció en su obra una lista de ciudades norteafricanas que están documentadas con anterioridad a la incorporación a Roma. Así pues, con anterioridad a la conquista romana ya existía una cierta tradición de vida urbana, más ligada a zonas costeras, pero también con su presencia en territorios del interior ${ }^{36}$.

Por otra parte, las urbes que la arqueología ha documentado en la época de la provincia romana (a partir de mediados del siglo I) reflejan el establecimiento del modelo de domus romana aristocrática ${ }^{37}$, así como una monumentalización del espacio urbano propia del mundo romano, quizás en este caso mostrando a las claras el influjo de la arquitectura militar ${ }^{38}$. El gran problema, sin duda, se encontraba en el escaso número de esas ciudades y de las regiones que las mismas centralizaban como era característico en el mundo romano, un puñado de las mismas en el momento mismo de la conquista romana tal y como refleja en ese momento Pomponio Mela ${ }^{39}$, situación

35 El proceso fue espléndidamente expuesto en su día a partir de las excavaciones en el Norte de Marruecos por parte de TARRADELL, M., «Sobre las raíces remotas de la Historia de Marruecos», Hespéris-Tamuda, 1, 1961, pp. 171-177; IDEM, «Las primeras civilizaciones de Marruecos», Cuadernos de la Biblioteca Española de Tetuán, 3, 1966, pp. 39-55. La novedad desde entonces estriba en que se ha podido detectar la mayor extensión del impulso urbanizador hacia el interior, a través de los ríos Loukous y Sebú; vid. por ejemplo LÓPEZ PARDO, F., «Los enclaves fenicios en el África noroccidental: del modelo de escalas náuticas al de colonización con implicaciones productivas», Gerión, 14, 1996, pp. 251288.

36 Aparte del caso de Volubilis, conocido por la arqueología, ALEX POLYHISTOR (en Esteban de Bizancio) nombra (junto a Zilil y Lixus) la ciudad de Gilda. De acuerdo con las investigaciones realizadas en este momento por Laurent Gallegharin y Mohamed Kebiri Alaoui, con mucha probabilidad, Gilda puede identificarse con Rirha, en el curso del río Beth; CALLEGARIN, L. y otros, "Les opérations archéologiques maroco-françaises de 2004 et 2005 à Rirha (Sidi Slimane, Maroc)", Mélanges de la Casa de Vélazquez, 36 (2), 2006, pp. 345-357.

37 Buen ejemplo al respecto es el de Volubilis; CARTOCCI, A., «Le domus di Volubilis», Annali deIla Facoltà di Lettere e Filosofia, 23, 2002, pp. 71-108, con toda la bibliografía anterior. Sobre el lujo de las casas es buena muestra la decoración con mosaicos; LIMANE, H., REBUFFAT, R. y DROCOURT, D., Volubilis, de mosaïque à mosaïque, Paris, 2000.

38 Vid. THOUVENOT, R., «L' urbanisme romain», op. cit.; LENOIR, M., «Le forum de Volubilis», en Los foros romanos de las provincias occidentales, Madrid, 1987, pp. 203-220; EUZENNAT, M. y HALLIER, G., "Les fórums de Tingitane. Observations sur I" influence de l' architecture militaire sur les constructions civiles de l' Occident romain», Antiquités Africaines, 22, 1986, pp. 73-103.

39 MELA III, 10, que en párrafo alterado por los copistas recogía a continuación una lectura difícil. En su edición de la Teubner en 1880, Frick restituyó Gilda, Volubilis, Prisciana, y así lo asumió ROGET, 
enormemente similar en las urbes documentadas por Plinio tres décadas después de la conquista romana ${ }^{40}$. No parece que la situación cambiara mucho con el desarrollo de la Historia de la provincia, en la que no aumentó sustancialmente el número ${ }^{41}$ : en el mejor de los casos unas 18 ciudades, con toda probabilidad una cifra inferior a ese número.

Esta cifra, entre 15 y 18 entidades urbanas, debe ponerse en relación con la Bética, una provincia profundamente romanizada, para obtener conclusiones comparativas. El territorio ocupado por Roma en la Tingitana era algo superior a la mitad del representado por la Bética romana. En ese espacio Plinio menciona 175 oppida, concepto que también aplica a algunas ciudades de Marruecos ${ }^{42}$. Este dato significa que la relación de ciudades por territorio en la Bética respecto a la Tingitana es más de cinco veces mayor. Esta extrema diferencia es la que marca el contraste entre un territorio muy urbanizado y otro escasamente urbanizado. En cualquier caso, es cierto que para la época de Claudio sabemos especificamente el estatus como colonias de cinco de esas entidades (Tingi, Zilil, Lixus, Babba y Banasa), expresamente por la epigrafía sabemos del título municipal de Volúbilis, pero con toda probabilidad ya tenían también esa categoría municipal otras dos, en concreto Sala y Rusadir. Del resto carecemos de datos de su estatus administrativo.

La segunda característica la centramos en que esas ciudades eran de unas pequeñas dimensiones. Así lo reflejaba de una forma expresa el propio Pomponio Mela, cuando decía de ellas parvas urbes. No hay mayor problema al respecto, pues sabemos que las ciudades hispanas también eran pequeñas, con la excepción de las capitales. Si comparamos los espacios urbanos, las ciudades de la Tingitana, a partir de la construcción de los recintos murados (una parte de ellos en la segunda mitad del siglo II), podemos observar que quizás la ciudad mayor era Zi${ }_{1 i{ }^{43}}$ con unas medidas difíciles de precisar (¿quizás unos 15.000 habitantes?). Para el recinto de Volubilis ${ }^{44}$ se ha aportado una cifra de 12.000 habitantes, y la misma asemeja un máximo. Los recintos de Tingif ${ }^{45}$ o de Lixus $^{46}$ no puede supo-

R., p. 29. Sin embargo, el texto alterado apunta a las dos primeras, pero la terminación Tania apunta mucho más a la ciudad de Tamuda. De acuerdo con ello, en la época de la conquista serían ciudades principales Rusgada (Rusadir=Melilla), Tamuda, Tingi, colonia (Zilil), Lixus, Sala, Gilda y Volubilis.

40 Por su parte PLINIO, NH. V, 2 y ss. confirma los casos de Rusadir, Tamuda (la cita en pasado), Tingi, Zilil, Lixus, Babba y Sala, en las que coincide con Mela pero añade Banasa.

41 En el mejor de los casos, las problemáticas Cobucla y Parietina en el litoral mediterráneo, nada seguros como ciudades; más claro parece el caso de Abila-Septem Fratres (Ceuta) que pudo alcanzar reconocimiento urbano; en la vía occidental del Itinerario de Antonino pueden añadirse Tabernis, Frigidis y Thamusida, y en el oriental Oppido Novo, Tremulis, Vopiscianis y Aquis Dacicis.

42 PLINIO, NH. III, 7

43 LENOIR, E., «La ville romaine de Zilil du I au IV siècle ap. J. C.», en L'Afrique Romaine, I siècle av. J. C,.- debut V siècle ap. J. C., Paris, 2005, pp. 65-76; EL-KHATIB, N. y LENOIR, M., «Le Maroc du Nord: le site de Zilil et sa région», en MONGNE, P. (Ed.), Archéologies : vingt ans de recherches françaises dans le monde, Paris, 2005.

44 PANETIER, J. L., Volubilis, une cité du Maroc Antique, Casablanca, 2002.

45 PONSICH, M., «Tanger Antique», ANRW, 10 (2), 1982, pp. 787-816; IDEM, «Origines et témoignages de l' histoire antique de Tanger», Actas Congreso Internacional El Estrecho de Gibraltar, vol. 1, Madrid, 1988, pp. 39-54.

46 TARRADELL, M., Lixus, Tetuán, 1959; PONSICH, M., «Lixus, les informations archéologiques», $A N R W, 10$ (2), 1982, pp. 817-849. 
nerse para ellos, por su extensión, cifras superiores a los 8.000-9.000 habitantes, y lo mismo puede decirse de colonia Iulia Valentia Banasa ${ }^{47}$. Y en todos esos casos estamos hablando de las ciudades principales. Naturalmente que existía también mucha población que habitaba el medio rural próximo, pero estos datos nos reflejan las limitaciones demográficas, muy fuertes, del Marruecos romano.

La tercera característica gira en torno a la sociedad documentada en las ciudades romanas. Sin ir más lejos en el análisis, se detecta una cierta diferencia entre la población de Tingi, una colonia romana, y la de Volubilis, un municipio. En la primera hay una mayor diversidad social representada en la epigrafía, mientras por el contrario en Volubilis la lista de los cargos públicos, de un lado, y también los propios homenajes públicos y privados, indican que la ciudad estaba al cargo de una oligarquía bastante estrecha ${ }^{48}$. El escaso dinamismo social se muestra, entre otros aspectos, en la escasa presencia de los libertos, que además es coherente con otra de las conclusiones del análisis epigráfico: el casi nulo desarrollo del modo de producción esclavista, que también suele utilizarse como un reflejo del grado de romanización ${ }^{49}$. Junto a ello en Volúbilis elementos religiosos parecen mostrar una fuerte perduración de componentes indigenistas que, si no son contradictorios, sí marcan un peso de la tradición local ${ }^{50}$.

Pero además, otro elemento de la sociedad muestra un aspecto muy significativo, nos referimos al enorme peso del estamento militar, tal y como podemos detectar de forma muy nítida en la epigrafía. El mismo es coherente con el propio hecho de que se asume que la Tingitana constituía una provincia con un contingente de tropas relativamente importante, que conocemos por toda una serie de campamentos militares establecidos en la provincia desde el Norte hasta el limes meridional ${ }^{51}$, así como por la colección de diplomas militares aparecidos, la más completa de todo el Imperio ${ }^{52}$. Este

47 THOUVENOT, R., Une colonie romaine de Maurétanie Tingitane : Valentia Banasa, Paris, 1941 ; LIMANE, H., ARHARBI, R. Y LENOIR, E., «Banasa», en MONGNE, P. (Ed.), pp. 237-238.

48 GOZALBES, E., «Notas sobre culto y sociología funeraria romana: el caso del municipio romano de Volubilis (Mauritania Tingitana), en MAYER, M. y GÓMEZ PALLARÉS, J. (Coords.), Religio Deorum. Actas del Coloquio internacional de epigrafía Culto y Sociedad en Occidente, Sabadell, 1994, pp. 295302; LEFEBVRE, S., «Hommages publics et histoire sociale: les Caecelii Caeciliani et la vie municipale de Volubilis (Maurétanie Tingitane)», Mélanges de la casa de Vélazquez, 28, 1992, pp. 19-36

49 El contraste entre el no desarrollo del esclavismo en la Tingitana, y su fuerte implantación en la Bética ya fue destacado por BLÁZQUEZ, J. M., «L' esclavage dans les exploitations agricoles de l' Hispania romaine», Mélanges de la Casa de Vélazquez, 8, 1972, pp. 636-642 (=Nuevos estudios sobre la romanización, Madrid, 1989, pp. 283-292).

50 El edificio más grande de la ciudad, el Templo B está dedicado a una divinidad indígena asimilada a Cronos-Saturno; MORESTIN, H., Le temple B de Volúbilis, Paris, 1980. Junto a ello la importancia del culto a la divinidad Aulisua, bien representada en la epigrafía; LENOIR, M., «Aulisua, dieu maure de la fecondité», Africa Romana, III, Sassari, 1988, pp. 295-302; BROUQUIER-REDDÉ, V., «De Saturne à Aulisua. Quelques remarques sur le panthéon de la Maurétanie Tingitane», Mélanges à la mémoire de Marcel Le Glay, Bruselas, 1994, pp. 154-164. Vid. También EL KHAYARI, A. Y BROUQUIER-REDDÉ, V., «Les monuments religieux de Maurétanie Tingitane», en MONGNE, P. (Ed.), pp. 239-240.

51 REBUFFAT, R., «L' implantation militaire romaine en Maurétanie Tingitane», Africa Romana, IV, Sassari, 1987, pp. 31-78; “L' Armée de la Maurétanie Tingitane», Mélanges de l'École Française de Rome, 110 (1), 1998, pp. 193-242.

52 Y que no cesan de aumentar; LENOIR, M., «Diplomes militaires inédites de Volubilis», Bulletin d' Archéologie Marocaine, 15, 1983-1984, pp. 213-224; PAPI, E., «Diplome militaire de Thamusida (Mau- 
peso importante del elemento militar, que en otros casos pasa mucho más desapercibido, es típico del medio que Leveau caracterizó como de una tierra de configuración colonial, característica que el propio desarrollo de época alto-imperial no disipó.

La cuarta característica que debemos destacar es la de la limitación en el país de las grandes obras de infraestructuras. Sin duda en Tingi debió existir un puerto importante, con sus instalaciones adecuadas, pues a través del mismo se producía la entrada y salida de los viajeros, y también de una parte importante de productos comerciales. También hoy conocemos mejor otro de los elementos más característicos del mundo romano, como son los importantes dispositivos de aprovisionamiento de agua, en especial para las ciudades, pero también para el suministro necesario en las numerosas fábricas de salazones de pescado existentes en el Norte de la provincia ${ }^{53}$.

Pero no hay evidencias de construcción de otros puertos importantes, más allá de posibles obras más modestas, ni de canales de regadío considerables, ni de presas importantes, al contrario de lo que ocurre en otras zonas del Magreb. Por el contrario, las vías de comunicación de esta provincia africana carecían en su gran mayoría de obras relevantes, no quedan evidencias de importantes puentes romanos lo que es muestra de su modestia, y sabemos en todo caso que los caminos no estaban calzados, es decir, eran simples pistas terreras, y de forma coherente con ello, no había inscripciones milliarias. Lo planteamos ya en su día ${ }^{54}$, y nada aparta de la evidencia la conclusión: la actitud de Roma al respecto es la más característica de una zona perfectamente subdesarrollada en relación con otras del Imperio ${ }^{55}$. Este hecho nos retrotrae a la conclusión principal alcanzada por Carcopino y expuesta con anterioridad: la fuerte limitación de la política romana en el territorio más occidental del magreb, insuficiente en esfuerzos para alcanzar una transformación más afectiva de una parte considerable de los habitantes.

\section{UNA SOCIEDAD DE FRONTERA: LAS TRIBUS INDÍGENAS}

La historiografía de la antigüedad comparó el África romana con el doble rostro de Jano: por un lado, la proclividad a la transformación, a la inclusión en las es-

\footnotetext{
ritania Tingitana), 31 décembre 133/134», ZPE, 142, 2003, pp. 257-265; ECK, W. Eck y PANGERL, A., «Weitere Militärdiplome für die mauretanischen Provinzen», ZPE, 162, 2007, pp. 235-247; DERKS, T., «A fragment of a new Roman military diploma for Mauretania Tingitana», Archaeologisches Korrespondenzblatt, 37 (2), 2007, pp. 257-269.

53 PONS PUJOL, L., «Los acueductos de Mauritania Tingitana. Estado de la cuestión», en LAGOSTENA, L. G., CAÑIZAR, J. L. y PONS, L. (Eds.), Aqvam perdvcendam cvravit. La captación, los usos y la administración del agua en las ciudades de la Bética y el Occidente romano, Cádiz, 2010, pp. 533542.

54 GOZALBES, E., Economía, passim.

55 En este sentido debemos remitir a los trabajos de DEMAN, A., «Rome et le sous-développement de l'Afrique», Revue des Études Anciennes, 81, 1979, pp.67-104; «Materiaux et réflexions pour servir a une étude du développement et du sous-développement dans les provinces de I" Empire Romain", ANRW, II (3), 1975, pp. 3-97.
} 
tructuras jurídicas, políticas, sociales, económicas y culturales de Roma, mostrada incluso con notable entusiasmo por parte de muchas elites; por el otro, el del rechazo a unas formas de vida consideradas extrañas, a unos ritos foráneos y mal comprendidos por determinados sectores de la población. La historiografía ha sido consciente de que la tensión o diferencia fundamental en el Norte de África radicó en el sedentarismo agrícola y el desarrollo de las formas de vida urbanas, frente al semi-nomadismo y al predominio de unas estructuras de carácter tribal. En relación con el propio Marruecos el geógrafo Pomponio Mela, a mediados del siglo I, daba una de sus características principales: hominum pars silvas frequentant, minus quam quos modo diximus, vagi; pars in urbibus agunt ${ }^{56}$.

Un característica especial viene determinada por aquello que la historiografía anglosajona ha definido como una «sociedad de frontera». Se trata de conceptualizar el contraste entre dos modos de vida, el «civilizado» y el caracterizado por comunidades con organización tribal. o del componente de carácter indígena, no asimilado a la vida urbana. Al final de cuentas, las «sociedades de frontera» a largo plazo están abocadas al choque, en la medida en la que sus soluciones no sean complementarias. Los elementos de cooperación en el terreno económico fueron muy predominantes en las primeras décadas posteriores a la conquista, con la explotación de recursos exóticos (marfil, madera de cidro), pero cuando éstos se agotaron se intensificaron los problemas.

Número muy limitado de ciudades, y además abundante población extra-urbana organizada en comunidades, o si se quiere en «reservas» (aunque en el caso de la Tingitana, éstas eran tan amplias que rebasaban ese posible concepto); Ptolomeo, justo un siglo después de la conquista, refleja ya la recuperación del medio indígena extra-urbano, con la mención de una gran cantidad de pueblos autóctonos en el territorio de vida tribal o semi-tribal ${ }^{57}$. De esta forma, podemos observar claramente la presencia de estas poblaciones indígenas o autóctonas en tierras no ocupadas por los agricultores sedentarios romanizados: zonas del litoral del Rif por los Socossii, en las zonas del interior de la costa del Estrecho (Anyera) por los Massaisulis, la zona montañosa del espinazo interior de Jbala por los Verbicae, y después otros pueblos menores, aunque los principales eran sin duda los Zegrenses, cerca de Banasa, los Baniubae de localización más imprecisa, y los Baquates, en todas las zonas de llanura desde Fez hacia el Norte y Este. Al Sur, y hasta el Atlas, los Macenitas como pueblo extremo y en buena parte externa gentes. En la zona costera, al Sur de Rabat, los Autololes (anterior-

56 MELA III, 10 ; ROGET, R., Le Maroc chez les auteurs anciens, Paris, 1923, p. 29 ; GOZALBES, E., «La descripción de Mauritania Tingitana en Pomponio Mela», Actas // Congreso Internacional El Estrecho de Gibraltar, vol. 2, Madrid, 1995, pp. 259-265.

57 PTOLOMEO IV, 5 ; HAMDOUNE, C., «Ptolémée et la localisation des tribus de Tingitane», Mélanges de l' École Française de Rome, 105 (1), 1993, pp. 241-289. Las «reservas» indígenas están bien documentadas por la epigrafía en otras zonas del África romana; CHAUSA, A. M., «Modelos de reservas de indígenas en el África romana», Gerión, 12, 1994, pp. 95-102. 
mente llamados Pharusios y Nigritas) que eran pueblos gétulos, no asimilados pero en relación con las autoridades romanas.

Los elementos y situaciones de conflicto, de «rebelión armada», quizás han sido algo exageradas en la historiografía más tradicional. Conviene por tanto el tener en cuenta, aunque sea de pasada, estas menciones. Pausanias nos habla de un fuerte problema en época de Antonino Pio, con los que parece que eran unos «moros» de más allá de las fronteras, en la zona del Atlas, unas poblaciones que de forma expresa se indica que eran de carácter nómada ${ }^{58}$. Así pues, no se trataba de un levantamiento de indígenas del interior, sino de la irrupción de pueblos de más allá de la zona de ocupación, con toda probabilidad esos Macenitas de los que antes hemos hablado ${ }^{59}$.

Por su parte, los Scriptores Historiae Augustae aportan unas citas muy genéricas, y que pueden interpretarse de formas diferentes en relación con acontecimientos de la época imperial; el episodio en el que se indica que Lucio Quieto sublatis gentibus Mauris, quos regebat, si además tenemos en cuenta la frase siguiente quia suspectus imperio fuerat, exarmavit ${ }^{6}$, nos está referiendo una sublevación mandada por el propio procurador, que al parecer aspiraba (o se sospechaba que aspiraba) al imperio, y por tanto no un levantamiento indígena. Aún y así, es cierto que el propio biógrafo señala en otro lugar que los pueblos controlados por Trajano (nationibus quas Traianus subegerat), entre ellos los moros, se levantaron contra la autoridad imperial61; y más adelante, cuando indica con la expresión Maurorum compressit, hay pocas dudas del sometimiento de los indígenas después de un levantamiento ${ }^{62}$.

La consecución en época de Antonino Pio de Mauros ad pacem postulandam coegit ${ }^{63}$, puede relacionarse con el mismo episodio citado con anterioridad; siguen quedando muy amplios márgenes a la interpretación en las citas de época de Marco Aurelio a que Mauri Hispaniae prope omnes vastarent ${ }^{64}$, o quod Baeticam Mauri populabuntur, en época de Cómodo se indica el sometimiento de los moros realizado por los legados del emperador ${ }^{65}$. Estas y otras citas reflejan problemas del ejército imperial con los moros, pero en un momento en el que la palabra «moro» había rebasado muy ampliamente el territorio de la antigua Tingitana; de hecho, la única referencia expresa a problemas en esta provincia se produce en época de Alejandro Severo ${ }^{66}$.

58 PAUSANIAS VIII, 43, 4. Con toda probabilidad estos moros nómadas del Atlas corresponden con el pueblo de los Macenitas, citados en esa zona por DION CASSIO LXXV, 13; DESANGES, J., Catalogue des tribus africains à l' Occident du Nil, Dakar, 1962. Es muy verosímil la identificación del nombre de los Macenitas con el de los Masmudas de la Edad Media.

59 GOZALBES, E., «Tumultos y Resistencia indígena en Mauretania Tingitana (siglo II)», Gerión, 20 (1), 2002, pp. 451-485.

60 SHA, Vita Hadriani, V, 8.

61 SHA, Vita Hadriani, V, $1,2$.

62 SHA, Vita Hadriani, XII, 7-8

63 SHA, Vita Antoninus Pius, V, 4. Un epígrafe de Sala, datado en el año 144, habla de los constantes ataques contra el territorio municipal por parte de grupos de bárbaros, y a los que el homenajeado M(arcus) Sulpicius Felix logró poner final.

64 SHA, Vita Marcus Antoninus, XXI, 1.

65 SHA, Vita Commodus, XIII, 5.

66 SHA, Vita Severus, LVIII, 1. 
En este sentido no parece raro que estas menciones se hayan observado desde dos perspectivas muy diferentes. Para unos estudiosos, los episodios de los SHA manifestarían la existencia de unos problemas militares constantes, unas fuertes rebeliones indígenas que obligarían a una presión constante para las autoridades romanas, en la línea de Cagnat, mantenida sin más, con pocas precisiones, por autores como Rachet o Sigman ${ }^{67}$. Por el contrario, otros investigadores han rebajado sensiblemente la importancia de estos incidentes, sobrevalorados por la propaganda, y que en su mayor parte corresponderían mucho más a actuaciones de policia que de ejército propiamente dicho ${ }^{68}$, y al propio hecho de que muchos de los problemas parecen en realidad motivados por la irrupción de pueblos del exterior ${ }^{69}$.

Las inscripciones latinas de Marruecos muestran la existencia de unos dirigentes tribales (príncipes o reyes) que son reconocidos por parte de Roma. Así la política seguida, y que se manifiesta en la documentación epigráfica, refleja un mútuo reconocimiento ${ }^{70}$. Los baquates, al igual que los restantes pueblos indígenas, eran objeto de derechos, puesto que a ellos se les aplicaba el iure gentis, tal y como se deduce claramente de un texto epigráfico de particular importancia, la Tabula Banasitana, en la cual en época de Marco Aurelio se concedía la ciudadanía romana a un princeps de los Zegrenses ${ }^{71}$. Destaca que en dos ocasiones, los baquates están unidos con otros pueblos de grandes dimensiones, entre los años 173-175 con los macenitas ${ }^{72}$, y entre 223-234 con los bavares $^{73}$, lo que demuestra la fortaleza de los mismos, capaces de formar confederaciones muy potentes, en un caso hacia el Sur, y en el segundo hacia el Este.

La epigrafía manifiesta algunos datos sobre la organización de las tribus, en lo que se refiere a su autoridad reconocida por Roma. Se trata, por otra parte, de una

67 RACHET, M., «Rome et les Berbères, un problème militaire d’ Auguste à Dioclétien, Bruselas, 1970 ; SIGMAN, The Role of the Indigenous Tribes in the Roman occupation of Mauritania Tingitana, Ann Arbor, 1976 ; IDEM, «The Romans and the Indigenous Tribes of Mauritania Tingitana», Historia, 26 (4), 1977, pp. 415-439.

68 FREZOULS, E., «Rome et la Maurétanie Tingitane: un constat déchec?» Antiquités africaines, 16, 1980, pp. 65-93; IDEM, «La résistance armée en Maurétanie de l' annexion à l' époque séverienne: un essai d' appréciation», Cahiers de Tunisie, 117-118, 1981, pp. 41-69; FÉVRIER, P. A., «A propos des troubles de Maurétanie» Zeitschrift für Papyrologie und Epigraphik 43, 1981, pp. 143-146.

69 EUZENNAT, M., «Les troubles de Maurétanie», Comptes Rendus de l'Académie des Inscriptions et Belles-Lettres, 1984, pp. 372-393

70 SHAW, B. D., «Autonomy and Tribute : mountain and plain in Mauritania Tingitana», Hommage à Jean Dresh, Paris, 1987, pp. 66-89.

71 EUZENNAT, M. y SESTON, W., «Un dossier de la chancellerie romaine, la Tabula Banasitana. Etude de diplomatique», Comptes Rendus de l' Académie des Inscriptions et Belles-Lettres, 1971, pp. 468-490 ; SHERWIN-WHITE, A., «The Tabula of Banasa and the Constitutio Antoniana», Journal of Roman Studies, 63, 1973, pp. 86-98.

72 Expresamente documentado en IAM, 2. Inscriptions Latines, no 384 : conlocut(us) / cum Vcmetio, prin/cipe gentium Ma/cennitum et Baqua/tium. Debe observarse que en la alianza los Macenitas aparecen mencionados en primer lugar.

73 Expresamente documentado en IAM, 2. Inscriptions Latines, no 402 : colloquiim/[cum... principe]/ gentis Bavarum et Baquatum. Nuevamente aquí los Bavares aparecen mencionados en primer lugar. Este pueblo estaba asentado en la Mauretania Cesariense. 
constante en la política africana del imperio, la que se ha definido como acantonamiento territorial de pueblos indígenas, junto al reconocimiento/colaboración de su autoridad. Si en otras zonas del Magrib las tribus indígenas fueron puestas bajo la autoridad de un prefecto (praefectus gentis), por el contrario, en la Tingitana las gentes tuvieron una mayor autonomía, bajo sus propios príncipes o régulos. Este hecho se debe a que no eran las ciudades y sus territorios los que empujaban hacia una reserva indígena, más bien eran los indígenas los que en lo territorial empujaban hacia zonas de reserva de los agricultores sedentarios.

Distinto es el caso, sin duda, de poblaciones externas, no controladas, que serán las que supongan un mayor peligro. Ya hemos aludido a los autololes, que es probable que fueran perdiendo importancia. No obstante, los pueblos próximos al Atlas van a suponer un peligro importante. Los sucesos acaecidos en momentos diversos, y que tuvieron su principal reflejo en época de Antonino Pio, van a tener su respuesta temporal; entre 173 y 175 aparentemente los romanos lograron poner a macenitas y baquates bajo la autoridad de un príncipe común llamado Ucmetius. Esta unión duró cierto tiempo, pero no dejó de ser muy efímera: en 180 los baquates ellos solos aparecen bajo el mandato del príncipe Aurelius Canartha.

Siendo plurales las formaciones tribales de la Tingitana, no cabe duda de que, con diferencia, autololes, baquates y macenitas fueron los que podían ocasionar un mayor volumen de preocupaciones para la administración romana. Pero los primeros sufrieron un proceso de decadencia, que condujo ya en el siglo IV al cambio de su nombre por el de Galaules, siendo los otros dos las referencias principales; en el Itinerario de Antonino se menciona la Mauritania de Tingi, indicando uno de los manuscritos que allí Bacavates et Macenites Barbari morantur. Los romanos siempre se preocuparon de pactar con los baquates, convirtiendo a sus reyes o príncipes en instrumentos de su política, mientras la relación con macenitas parece que fue bastante más conflictiva.

Curiosamente, el mejor modelo de la situación romanizados/grupos indígenas es justamente el propio Marruecos medieval. Cuando en el siglo $\mathrm{X}$ el geógrafo oriental Ibn Hawqal describe la situación de los beréberes en el Sur de Marruecos señala que frente a las ciudades, establecidas en la zona, llevaban una vida más pobre, aunque algunos grupos sobrevivían mejor gracias a la ganadería. Otros grupos utilizaban el país como pastos y autorizaban el acceso a terrenos cultivables y a puntos de agua para los camellos y también para los ganados ${ }^{74}$. Estas situaciones, aunque desplazadas hacia el Sur, parecen dibujar el estado de las cosas en la antigua Mauretania Tingitana.

Roma convivió con el mundo tribal, a partir de un reparto de territorios. Pero esta sociedad de frontera tenía en sus propias limitaciones una señal de caducidad. El trasvase desde el medio tribal apenas se produjo, Roma no superó los lí-

74 Al respecto, GOZALBES BUSTO, G. y GOZALBES, E., «El elemento tribal en Marruecos: de la romanización a la arabización», Homenaje al Profesor José María Fórneas Besteiro, vol. 2, Granada, 1995, pp. 767-778. 
mites que la civilización clásica había alcanzado antes de su conquista del país. Mantuvo una política de control y apartamiento. La no integración de los indígenas se mantuvo, y aparentemente constituye una actitud secular. En el siglo X el Emir de Fez Muhammad ibn Idris dirigió una carta a Abd-ar-Rahman III al-Nasir de Córdoba, en la que se refería a la actuación de su antepasado Idris I en los siguientes términos: cuando nuestro abuelo vino a los beréberes y les pidió asilo, se lo dieron y reconocieron su derecho, dándole una asignación sobre el país, a cambio de hacer de arbitro entre ellos, sinq ue tuviera sobre ellos mando. Él murió, dejándonos a sus descendientes que ocupamos su lugar e hicimos como él, pues los beréberes siguen actualmente en los mismos antiguos términos con nosotros, de manera que si queremos imponer autoridad, nos rehuyen y se nos encastillan, por lo que unas veces los combatimos y otras los halagamos, sin intentar en ningún momento gobernarlos y refrenar su agresividad ${ }^{75}$. En gran manera, estas mismas expresiones podrían haber sido pronunciadas por los procuradores romanos de la Tingitana. Más allá de la discusión teórica sobre el «eterno beréber», lo cierto es que la perduración de este tipo de formas de vida, y de resistencia al control por parte de un poder central, aparece como uno de los fenómenos profundos de la Historia de Marruecos.

\section{EL BALANCE FINAL}

El análisis de la Mauritania Tingitana se inserta en el ámbito general de estudio del África romana. Muchas de las problemáticas de la provincia occidental son comunes, o muy similares, a las del conjunto. Aún y así, es indudable que la provincia de la Tingitana encierra una fuerte peculiaridad: no es enteramente diferente al resto, pero las trazas de urbanización son menores, mientras las características de semi-nomadismo o de tribalismo son mayores. Así pues, se trataba de un territorio en el que la balanza con toda probabilidad guardaba menos equilibrio que en el resto: muchas menos ciudades y, en consecuencia, proporción mayor de grupos tribales. Y una balanza, ciertamente no sólo en ella sino en parte de la cercana Argelia también, que se inclinó de forma definitiva hacia el lado del rechazo final a la romanitas ${ }^{76}$.

Esta situación misma, este sólido desequilibrio en contra de las transformaciones romanas, ni mucho menos se dió en todas partes, aunque las citas de Ammiano Marcelino son suficientemente expresivas de cómo en el siglo IV los pueblos refractarios al dominio romano, con estructuras socio-familiares diferentes, ocupaban unas posiciones muy importantes en otros territorios muy extensos de Ar-

75 IBN HAYYAN, Al-Muqtabis, $V$, trad. de CORRIENTE, F. y VIGUERA, M. J.,, Zaragoza, 1981, p. 220

76 GOZALBES, E. Y GONZÁLEZ, I., «De la Romanitas a la romanización: propaganda y poder político", en BRAVO, H. y GONZÁLEZ, R. (Eds.), Toga y daga. Teoría y praxis de la política en Roma, Madrid, 2010, pp. 33-47. 
gelia $^{77}$. Y también Abdallah Laroui, uno de los mejores pensadores magrebíes del siglo XX, argumentó en su Historia del Magreb que el rechazo a la romanitas constituyó, para muy amplios grupos indígenas, la respuesta necesaria al bloqueo y marginalidad a la que los sometieron la política de Roma, y la propia acción de las elites rectoras de la sociedad mauritano-romana ${ }^{78}$. Baste indicar que, sin duda, tampoco es que Roma pudiera llegar mucho más allá, puesto que los gastos de ocupación rebasaban los beneficios de la misma, pese a la evidente expansión económica y productiva (agrícola y pesquera en especial) de la primera mitad del siglo III.

No obstante, también la crisis del siglo III fue lo suficientemente potente como para poner en cuestión las situaciones. Es cierto que en la Tingitana, al igual que acontece en el África Proconsular ${ }^{79}$, el máximo de la explotación económica se detecta en torno al año 230 y en Volúbilis las grandes obras de monumentalización, además del llamado «Palacio de Gordiano», son de esta época. Sin embargo, los acontecimientos de la pavorosa crisis, si bien con retraso, también llegaron a la Mauretania Tingitana, y son muy evidentes en cuanto a inestabilidad y recesión ${ }^{80}$. La misma no significó una ruptura definitiva, pero sí afianzó y probablemente recreció los topes que la política romana no podía superar: escasa ganancia de la posesión de un territorio, y escasa impregnación del tejido extra-urbano.

En el año 285 la administración romana, siguiendo la política aplicada por parte de Diocleciano en otras zonas, evacuó una parte de la provincia ${ }^{81}$, entregándola sin duda para su control al pueblo federado de los Baquates ${ }^{82}$. La nueva provincia, adscrita a la diocesis Hispaniarum, quedó reducida básicamente a la península tingitana, y en la misma se detecta la reactivación del llamado «renacimiento constantiniano». En las primeras décadas del siglo V, probablemente en el mismo 429 con el paso de los vándalos a través del Estrecho de Gibraltar,

77 SANTOS YANGUAS, N., «La resistencia de las poblaciones indígenas norteafricanas a la romanización en la segunda mitad del siglo IV d. C.», Hispania, 142, 1979, pp. 257-300.

78 LAROUI, A., L' Histoire du Maghreb. Un essai de synthèse, Paris, 1970.

79 RAMIREZ SÁDABA, J. L., Gastos suntuarios y recursos económicos de los grupos sociales del África romana, Oviedo, 1981.

80 GOZALBES, E., “La crisis del siglo III en Mauritania Tingitana», en CRESPO, S. y ALONSO, A. (Eds.), Scripta Antiqva in Honorem Angel Montenegro Duque et Jose María Blázquez Martínez, Valladolid, 2002, pp.693-701.

81 La tesis del repliegue romano, con abandono de Volubilis, Thamusida o Banasa, fue formulado en su día por parte de CARCOPINO, J., «La fin du Maroc romain», Mélanges d' Archéologie et d’ Histoire de l' Ecole Française de Rome, 1940, pp. 349-448. Las investigaciones posteriores no han hecho otra cosa que confirmar que estas ciudades no fueron deshabitadas pero su población dejó, en buena parte, de seguir los moldes de la vida romana, por ejemplo en la economía monetaria y objetos de consumo. El número de monedas del siglo IV es ridículo respecto al de otras épocas, o al que aparece en el Norte de Marruecos, que siempre representa un máximo.

82 El pueblo de los Baquates aparece como el principal del territorio, y que tenía sus territorios en las zonas centrales de Marruecos no ocupadas por los romanos, en torno a Fez. En la epigrafía volubilitana aparece desde la primera mitad del siglo II firmando y confirmando paces con el procurador provincial, señalando que sin duda era el principal del territorio. La tesis de que recibió el reconocimiento de «pueblo federado» parece coherente, aunque no es del todo segura, puesto que la expresión del epígrafe puede referise a pax foederata. En cualquier caso, como antes reflejamos, en una nota al inicio de un manuscrito del Itinerario Antonino se indica que en la Mauritania Tingitana Baquates et Macenitas barbari morantur, con lo que se confirma el papel predominante de los Baquates. 
Roma perdió totalmente el control de los restos de la provincia Tingitana, en la península Noroeste, controlada hasta ese momento por los soldados limitanei, bajo la autoridad del praeses ${ }^{83}$. La historiografía ha dado por supuesto, con plena lógi$\mathrm{ca}$, que en esos momentos el balance de la pugna estaba ya decidido de forma aplastante en contra de la romanización.

Por el contrario, una Tesis Doctoral relativamente reciente, ha planteado puntos de vista diferentes. El estudio de Noé Villaverde ha recogido de forma muy completa toda la documentación de épocas bastante amplias, intentando probar la pujanza de las estructuras romanas en Marruecos hasta épocas muy tardías. La principal tesis apuntada por el autor es que en el momento de la llegada de los árabes, a finales del siglo VII, los elementos de romanización continuaban vigentes en este país de una forma muy sólida ${ }^{84}$. Naturalmente en el conocimiento histórico se avanza mediante la formulación de tesis, y mediante la contrastación de las mismas, pero más allá del valor de esta aportación a nuestro juicio la conclusión anterior, contraria a todas las opiniones hasta ahora expresadas, es muy difícilmente mantenible.

En efecto, Gilbert-Charles Picard defendió su punto de vista a partir no solamente del esplendor e intensidad de la romanización en Tunez, sino también del hecho de que Roma tuvo continuadores. El Reino vándalo en una buena parte mantuvo las estructuras romanas, como ha documentado muy bien la arqueología y la documentación representada por las Tablettes Albertin ${ }^{35}$; por otra parte, con posterioridad en el territorio también se produjo la ocupación por parte de los bizantinos, que volvieron a consolidar las posiciones de predominio de la vida urbana en la provincia nucleada en torno a Cartago. Este hecho es fuertemente diferencial, más allá de una hipotética influencia vándala en la Tingitana, y de una ocupación circunscrita a Septem (Ceuta) y quizás algún punto costero más.

Como indicamos antes, el balance contrario a la romanización estaba ya bastante bien despejado con mucha anterioridad a esa época. Y fue así en primer lugar por las propias y fuertes limitaciones de la política romana en la Tingitana, como Carcopino supo muy bien leer. No creemos necesario ahora insistir en este aspecto, si bien los datos parecen clarificadores, más allá naturalmente de algunos o bastantes éxitos de Roma, como fue el mantenimiento de su control en el país durante muchos siglos. Los grupúsculos de romanidad que perdurarán, por ejemplo en torno a la iglesia de Tingi, o los Roumis de Volúbilis del siglo VII, no pueden ni deben ser ocultados, pero son ejemplos minoritarios y en gran parte anecdóticos. Sin duda, el Islam se adaptó mejor a las condiciones del mundo tribal de lo que pudo hacerlo Roma.

83 NOTITIA DIGNITATUM, Occ. XXVI. La distribución de guarniciones con soldados limitanei, aunque no eran los únicos en el territorio, muestra una fuerte voluntad de ocupación y control, todas ellas en la península tingitana (con la excepción de Sala=Rabat en situación de presidio).

84 VILLAVERDE, N., Tingitana en la Antigüedad Tardía (siglos III.VII). Autoctonía y romanidad en el extremo Occidente del Mediterráneo, Madrid, 2001.

85 Vid. por ejemplo la monografía de GIL EGEA, M. E., África en tiempo de los vándalos: continuidad y mutaciones de las estructuras socio-políticas romanas, Alcalá de Henares, 1999. 
